\title{
A Numerical Method of the Euler-Bernoulli Beam with Optimal Local Kelvin-Voigt Damping
}

\author{
Xin Yu, ${ }^{1,2}$ Zhigang Ren, ${ }^{2}$ Qian Zhang, ${ }^{1}$ and Chao $\mathrm{Xu}^{2}$ \\ ${ }^{1}$ Laboratory of Information \& Control Technology, Ningbo Institute of Technology, Zhejiang University, Ningbo 315100, China \\ ${ }^{2}$ The State Key Laboratory of Industrial Control Technology and Institute of Cyber-Systems \& Control, \\ Zhejiang University, Hangzhou 310027, China
}

Correspondence should be addressed to Chao Xu; cxu@zju.edu.cn

Received 12 February 2014; Accepted 28 May 2014; Published 23 June 2014

Academic Editor: Magdy A. Ezzat

Copyright (c) 2014 Xin Yu et al. This is an open access article distributed under the Creative Commons Attribution License, which permits unrestricted use, distribution, and reproduction in any medium, provided the original work is properly cited.

\begin{abstract}
This paper deals with the numerical approximation problem of the optimal control problem governed by the Euler-Bernoulli beam equation with local Kelvin-Voigt damping, which is a nonlinear coefficient control problem with control constraints. The goal of this problem is to design a control input numerically, which is the damping and distributes locally on a subinterval of the region occupied by the beam, such that the total energy of the beam and the control on a given time period is minimal. We firstly use the finite element method (FEM) to obtain a finite-dimensional model based on the original PDE system. Then, using the control parameterization method, we approximate the finite-dimensional problem by a standard optimal parameter selection problem, which is a suboptimal problem and can be solved numerically by nonlinear mathematical programming algorithm. At last, some simulation studies will be presented by the proposed numerical approximation method in this paper, where the damping controls act on different locations of the Euler-Bernoulli beam.
\end{abstract}

\section{Introduction}

Let $T, L>0$ be two positive constants. We denote by $Q$ the product set $(0, L) \times(0, T)$. Consider a nonhomogeneous clamped elastic beam of length $L$, where one segment of the beam is made of a viscoelastic material with Kelvin-Voigt constitutive relation. By the Kirchhoff hypothesis, neglecting the rotatory inertia, the transversal vibration of the beam can be described by the following equation and boundary-initial conditions:

$$
\begin{gathered}
\rho \ddot{w}+\left(q w^{\prime \prime}+D \dot{w}^{\prime \prime}\right)^{\prime \prime}=f, \quad(x, t) \in Q, \\
w(0, t)=w(L, t)=w^{\prime}(0, t)=w^{\prime}(L, t)=0, \\
w(x, 0)=w_{0}(x), \quad \dot{w}(x, 0)=w_{1}(x),
\end{gathered}
$$

where $w$ represents transversal displacement of the beam, $w_{0}, w_{1} \in L^{2}(0, L)$ are given initial data, and the notations $\dot{w}$ and $w^{\prime}$ denote the derivatives with respect to the temporal variable and the spatial variable of $w$, respectively. Here $f \in$ $L^{2}(Q)$ is an external applied distributed force, $\rho$ is the linear mass density of the beam material, $q$ is the flexural rigidity, and $D$ is the Kelvin-Voigt damping coefficient. In this paper, we assume $\rho, q \in L^{\infty}(0, L), D \in L^{\infty}(Q)$, and $\rho, q \geq c>0$ for $x \in(0, L)$ for some constant $c$. we say that the Kelvin-Voigt damping is globally distributed if the damping coefficient $D \geq c>0$ on $[0, L]$; we say it is locally distributed if $D \geq c>0$ only on some subinterval of $[0, L]$ and $D=0$ elsewhere.

Smart materials such as shape memory alloys and piezoceramics [1-3] have been applied for active vibration control of elastic structures. Accordingly, one can introduce control terms to the elastic systems such as the damping coefficients and Young's moduli as well. Magnetorheological (MR) dampers [4-7] are one of the most promising new actuation mechanisms that use MR fluids to provide variable damping actuation for active control of structures. Because of their mechanical simplicity, high dynamic range, low power requirements, large force capacity, and robustness, these devices have been shown to mesh well with application demands and constraints to offer an attractive control method to structural vibration. 
In this paper, we will study the Euler-Bernoulli beam equation with optimal local Kelvin-Voigt damping. To be more specific, let $\omega \in[0, L]$ be a subinterval and let $\chi_{\omega}$ be the characteristic function of $\omega$, that is,

$$
\chi_{\omega}(x)= \begin{cases}1, & x \in \omega \\ 0, & \text { otherwise }\end{cases}
$$

We define

$$
\mathcal{U}=\left\{u(\cdot) \in L^{\infty}(0, T) \mid R_{1} \leq u(t) \leq R_{2} \text { for a.e. } t \in[0, T]\right\}
$$

and assume the damping coefficient $D$ has the following form:

$$
D(x, t)=\chi_{\omega}(x) u(t), \quad(x, t) \in Q
$$

where $R_{1}, R_{2} \in \mathbb{R}$ are two fixed constants and $u \in \mathcal{U}$ is a control function.

Let $V=H_{0}^{2}(0, L)$ with the norm

$$
\|w\|_{V}=\left(\int_{0}^{L} q(x)\left|w^{\prime \prime}(x)\right|^{2} d x\right)^{1 / 2}, \quad \forall w \in V
$$

and $H=L_{\rho}^{2}(0, L)$ with the norm

$$
\|v\|_{H}=\left(\int_{0}^{L} \rho(x)|v(x)|^{2} d x\right)^{1 / 2}, \quad \forall v \in H .
$$

Define $\mathscr{H}=V \times H$ with the norm

$$
\left\|\left(\begin{array}{c}
w \\
v
\end{array}\right)\right\|_{\mathscr{H}}=\left(\|w\|_{V}^{2}+\|v\|_{H}^{2}\right)^{1 / 2}, \quad \forall\left(\begin{array}{c}
w \\
v
\end{array}\right) \in \mathscr{H} .
$$

Then, $\mathscr{H}$ is Hilbert space and the energy of the beam at time $t$ is

$$
\begin{aligned}
\left\|\left(\begin{array}{c}
w(t) \\
\dot{w}(t)
\end{array}\right)\right\|_{\mathscr{H}}^{2}= & \int_{0}^{L} q(x)\left|w^{\prime \prime}(x, t)\right|^{2} d x \\
& +\int_{0}^{L} \rho(x)|\dot{w}(x, t)|^{2} d x,
\end{aligned}
$$

where $w$ is the solution of (1). The optimal control problem that we will study is formulated as follows:

$$
\begin{gathered}
(\mathrm{OCP}) \min _{u \in \mathscr{U}}\left\{J(u)=\frac{1}{2} \int_{0}^{T}\left\|\left(\begin{array}{c}
w(t) \\
\dot{w}(t)
\end{array}\right)\right\|_{\mathscr{H}}^{2} d t\right. \\
\left.+\frac{1}{2} \int_{0}^{T}|u(t)|^{2} d t\right\}
\end{gathered}
$$

subject to the controlled equation (1), where $w$ is the solution of (1). Throughout the paper, we will omit the notations $t$ or $x$ in the functions of $t$ or $x$ in the case that there is no risk to make any confusion.

Due to the importance from both perspectives of mathematics and applied science, the control problem of various beam equations has been considered by many researchers [810]. The study of the Euler-Bernoulli beam is one of the most active research topics in control theory. In [11], the authors consider the vibration of the Euler-Bernoulli beam with Kelvin-Voigt damping distributed locally on any subinterval of the region occupied by the beam. By making use of the frequency domain method and the multiplier technique, they prove that the semigroup associated with the equation for the transversal motion of the beam is exponentially stable. In [12], the author studies the basis property and the stability of a distributed system described by a nonuniform EulerBernoulli beam equation under linear boundary feedback control. The Riesz basis property is presented and the exponential stability is concluded. In [13], stabilization of EulerBernoulli beam by means of the pointwise feedback force is considered. Both uniform and nonuniform energy decay may occur, which depend on the boundary conditions. There are some other related papers about the studies of EulerBernoulli beam equations [14-17].

In this paper, we will study the numerical approximation of the optimal control problem (OCP), which is a nonlinear bilinear control problem with control constraint. Bilinear control problems are already studied by many researchers [18-20]. In our paper, we want to design a damping control numerically, which acts on local interval of the beam, such that the total energy of the beam and the control on a given time period is minimal. It appears that little work has been done on numerical methods for this problem. By the standard finite element method (FEM), problem (OCP) was firstly approximated by an optimal control problem $\left(\mathrm{OCP}^{h}\right)$ governed by a system of ordinary differential equations. Then, using the control parameterization method [21], we will approximate the finite-dimensional problem by another suboptimal problem $\left(\mathrm{OCP}_{d}^{h}\right)$, which is a standard optimal parameter selection problem and can be solved numerically by nonlinear mathematical programming algorithm. At last, some simulation studies will be presented by the numerical method proposed in this paper.

\section{The Semidiscrete Approximation by FEM}

In this section, we will approximate the original optimal control problem (OCP) with FEM method. Noting that (1) involves the spatial derivative of four orders, the conforming FEM space should belong to $H^{2}(0,1)$. Consider the interval domain $[0, L]$. The triangulation $\mathscr{T}^{h}$ of $[0, L]$ divides $[0, L]$ into a finite number of subintervals $I_{j}=\left[x_{j-1}, x_{j}\right], j=$ $1,2, \ldots, N+1$, using the grid points:

$$
0=x_{0}<x_{1}<\cdots<x_{N}<x_{N+1}=L,
$$

where we will call $I_{j}=\left[x_{j-1}, x_{j}\right]$ the $j$ th element and $h_{j}=$ $x_{j}-x_{j-1}$ the size of this element. The discretization parameter $h$ is the maximum size of all $h_{j}, j=1,2, \ldots, N+1$. Associated with every triangulation $\mathscr{T}^{h}$, we define a finite-dimensional space as follows:

$$
\begin{aligned}
& V^{h}=\left\{v^{h} \in C^{1}[0, L] \mid\right. \\
& v^{h} \in P_{3}\left(I_{j}\right) \text { for } j=1,2, \ldots, N+1, \\
& \left.v^{h}(0)=v^{h}(L)=\left(v^{h}\right)^{\prime}(0)=\left(v^{h}\right)^{\prime}(L)=0\right\},
\end{aligned}
$$


where $P_{3}\left(I_{j}\right)$ is the space of all polynomials of degree less than or equal to 3 over the subinterval $I_{j}$. Obviously, we have $V^{h} \in$ $H_{0}^{2}(0, L)$. Thus, we can write

$$
V^{h}=\operatorname{span}\left\{\phi_{i}, \mid i=1,2, \ldots, 2 N\right\},
$$

where, for $i=1,2, \ldots, N$,

$$
\phi_{i}\left(x_{k}\right)=\delta_{i k}, \quad \phi_{i}^{\prime}\left(x_{k}\right)=0, \quad \forall k=0,1, \ldots, N+1,
$$

and, for $i=N+1, N+2, \ldots, 2 N$,

$$
\phi_{i}\left(x_{k}\right)=0, \quad \phi_{i}^{\prime}\left(x_{k}\right)=\delta_{i-N, k}, \quad \forall k=0,1, \ldots, N+1 .
$$
setting

Define a bilinear form $(\cdot, \cdot)_{\rho}$ over $L_{\rho}^{2}(0, L) \times L_{\rho}^{2}(0, L)$ by

$$
(f, g)_{\rho}=\int_{0}^{L} \rho(x) f(x) g(x) d x, \quad \forall f, g \in L_{\rho}^{2}(0, L) .
$$

Define another two bilinear forms $a_{q}(\cdot, \cdot)$ and $a_{\omega}(\cdot, \cdot)$ over $H_{0}^{2}(\Omega) \times H_{0}^{2}(\Omega)$ by setting

$$
\begin{aligned}
& a_{q}(f, g)=\int_{0}^{L} q(x) f^{\prime \prime}(x) g^{\prime \prime}(x) d x, \quad \forall f, g \in H_{0}^{2}(\Omega), \\
& a_{\omega}(f, g)=\int_{\omega} f^{\prime \prime}(x) g^{\prime \prime}(x) d x, \quad \forall f, g \in H_{0}^{2}(\Omega),
\end{aligned}
$$

respectively. Obviously, the two bilinear forms $(\cdot, \cdot)_{\rho}$ and $a_{q}(\cdot, \cdot)$ are the inner products of $L_{\rho}^{2}(0, L)$ and $H_{0}^{2}(\Omega)$, respectively. Then, the finite element approximation of (1) consists in finding $w^{h}(t)=w^{h}(\cdot, t)$, which belongs to $V^{h}$ for $t \in[0, T]$, and satisfies

$$
\begin{gathered}
\left(\ddot{w}^{h}(t), v^{h}\right)_{\rho}+a_{q}\left(w^{h}(t), v^{h}\right)+u(t) a_{\omega}\left(\dot{w}^{h}, v^{h}\right) \\
=\left(f(t), v^{h}\right) \\
\forall v_{h} \in V^{h}, \quad 0<t \leq T, \\
w^{h}(0)=w_{0}^{h}, \quad \dot{w}^{h}(0)=w_{1}^{h},
\end{gathered}
$$

where $(\cdot, \cdot)$ denotes the standard inner product of $L^{2}(0, L)$ and $w_{0}^{h}, w_{1}^{h} \in V^{h}$ are the proper approximations of $w_{0}, w_{1}$ on $V^{h}$. In the following, we write

$$
\begin{gathered}
w^{h}(x, t)=\sum_{j=1}^{2 N} X_{j}(t) \phi_{j}(x), \\
w^{h}(x, 0)=w_{0}^{h}(x)=\sum_{j=1}^{2 N} X_{0 j} \phi_{j}(x), \\
\dot{w}^{h}(x, 0)=w_{1}^{h}(x)=\sum_{j=1}^{2 N} Y_{0 j} \phi_{j}(x) .
\end{gathered}
$$

Substituting (19) into (18) and taking $v_{h}=\phi_{i}$ yield that

$$
\begin{aligned}
\sum_{j=1}^{2 N}\left(\phi_{i}, \phi_{j}\right)_{\rho} \ddot{X}_{j}(t)+\sum_{j=1}^{2 N} a_{q}\left(\phi_{i}, \phi_{j}\right) X_{j}(t) \\
+u(t) \sum_{j=1}^{2 N} a_{\omega}\left(\phi_{i}, \phi_{j}\right) \dot{X}_{j}(t)=\left(f(t), \phi_{i}\right) .
\end{aligned}
$$

Moreover, in this paper, we take $w_{0}^{h}, w_{1}^{h}$ as the $L_{\rho}^{2}$-projection approximations of $w_{0}, w_{1}$ on $V^{h}$; that is,

$$
\left(w_{0}^{h}, v^{h}\right)_{\rho}=\left(w_{0}, v^{h}\right)_{\rho}, \quad\left(w_{1}^{h}, v^{h}\right)_{\rho}=\left(w_{1}, v^{h}\right)_{\rho}, \quad \forall v^{h} \in V^{h} .
$$

Then, substituting (20) into (22) and taking $v_{h}=\phi_{i}$ yield that

$$
\sum_{j=1}^{2 N}\left(\phi_{i}, \phi_{j}\right)_{\rho} X_{0 j}=\left(w_{0}, \phi_{i}\right)_{\rho}, \quad \sum_{j=1}^{2 N}\left(\phi_{i}, \phi_{j}\right)_{\rho} Y_{0 j}=\left(w_{1}, \phi_{i}\right)_{\rho} .
$$

Define

$$
\begin{gathered}
X(t)=\left[X_{i}(t)\right]_{2 N \times 1}, \quad M=\left[\left(\phi_{i}, \phi_{j}\right)_{\rho}\right]_{2 N \times 2 N}, \\
K=\left[a_{q}\left(\phi_{i}, \phi_{j}\right)\right]_{2 N \times 2 N^{\prime}}, \quad R=\left[a_{\omega}\left(\phi_{i}, \phi_{j}\right)\right]_{2 N \times 2 N}, \\
X_{0}=\left[X_{0 i}\right]_{2 N \times 1}, \quad Y_{0}=\left[Y_{0 i}\right]_{2 N \times 1}, \\
F(t)=\left[\left(f(t), \phi_{i}\right)\right]_{2 N \times 1}, \quad \eta=\left[\left(w_{0}, \phi_{i}\right)_{\rho}\right]_{2 N \times 1}, \\
\xi=\left[\left(w_{1}, \phi_{i}\right)_{\rho}\right]_{2 N \times 1} .
\end{gathered}
$$

Thus, by (21) and (23), we can obtain the following system of controlled ordinary differential equations:

$$
\begin{gathered}
M \ddot{X}(t)=-K X(t)-u(t) R \dot{X}(t)+F(t), \\
X(0)=X_{0}, \quad \dot{X}(0)=Y_{0},
\end{gathered}
$$

where $X_{0}=M^{-1} \eta, Y_{0}=M^{-1} \xi$. Let

$$
\dot{X}=Y, \quad Z=\left(\begin{array}{l}
X \\
Y
\end{array}\right) .
$$

We define

$$
\begin{aligned}
G(t, Z(t), u(t))= & \left(\begin{array}{cc}
0 & I \\
-M^{-1} K & -u(t) M^{-1} R
\end{array}\right) Z(t) \\
& +\left(\begin{array}{c}
0 \\
M^{-1} F(t)
\end{array}\right) .
\end{aligned}
$$

Then (25) can be rewritten as

$$
\begin{gathered}
\dot{Z}(t)=G(t, Z(t), u(t)), \\
Z(0)=Z_{0},
\end{gathered}
$$

where $Z_{0}=\left(\begin{array}{c}X_{0} \\ Y_{0}\end{array}\right)$. 
By (19), a direct computation yields

$$
\begin{aligned}
\left\|w^{h}(t)\right\|_{V}^{2} & =a_{q}\left(w^{h}(t), w^{h}(t)\right) \\
& =\sum_{i=1}^{2 N} \sum_{j=1}^{2 N} a_{q}\left(\phi_{i}, \phi_{j}\right) X_{i}(t) X_{j}(t)=(X, K X)_{\mathbb{R}^{2 N}},
\end{aligned}
$$

where $(\cdot, \cdot)_{\mathbb{R}^{2 N}}$ denotes the inner product of $\mathbb{R}^{2 N}$ and $V=$ $H_{0}^{2}(0, L)$. Similarly, we have

$$
\begin{aligned}
\left\|\dot{w}^{h}(t)\right\|_{H}^{2} & =\left(\dot{w}^{h}(t), \dot{w}^{h}(t)\right)_{\rho} \\
& =\sum_{i=1}^{2 N} \sum_{j=1}^{2 N}\left(\phi_{i}, \phi_{j}\right)_{\rho} \dot{X}_{i}(t) \dot{X}_{j}(t)=(Y, M Y)_{\mathbb{R}^{2 N}},
\end{aligned}
$$

where $H=L_{\rho}^{2}(0, L)$. Define

$$
\Pi=\left(\begin{array}{cc}
K & 0 \\
0 & M
\end{array}\right)
$$

Then, it follows from (29), (30), and (31) that

$$
\begin{aligned}
J(u) & \approx J^{h}(u) \\
& =\frac{1}{2} \int_{0}^{T}\left\|\left(\begin{array}{c}
w^{h}(t) \\
\dot{w}^{h}(t)
\end{array}\right)\right\|_{\mathscr{H}}^{2} d t+\frac{1}{2} \int_{0}^{T}|u(t)|^{2} d t \\
& =\frac{1}{2} \int_{0}^{T}(Z(t), \Pi Z(t))_{\mathbb{R}^{4 N}} d t+\frac{1}{2} \int_{0}^{T}|u(t)|^{2} d t .
\end{aligned}
$$

Thus, by combining (28) and (32), the semidiscrete approximation of problem (OCP) is formulated as follows:

$$
\left(\mathrm{OCP}^{h}\right) \min _{u \in \mathscr{U}} J^{h}(u)
$$

subject to (28).

\section{Piecewise-Constant Control Approximation}

In general, problem $\left(\mathrm{OCP}^{h}\right)$ cannot be solved analytically. Using the control parameterization method, which has been successfully applied to provide numerical solutions for a wide variety of practical optimal control problems [2124], we will approximate problem $\left(\mathrm{OCP}^{h}\right)$ by a standard optimal parameter selection problem. This method involves approximating the control function by a piecewise-constant function with possible discontinuities at a set of preassigned switching points, which produces an approximation problem such that the solution of this approximation is a suboptimal solution to problem $\left(\mathrm{OCP}^{h}\right)$.

Let $\tau_{k}, k=0,1, \ldots, d$, be prefixed time knot points satisfying

$$
0=\tau_{0}<\tau_{1}<\tau_{2}<\cdots<\tau_{d}=T
$$

With piecewise-constant basis functions, the control input $u$ for the problem $\left(\mathrm{OCP}^{h}\right)$ is approximated over the $k$ th control subinterval $\left[\tau_{k-1}, \tau_{k}\right)$ as follows:

$$
u(t) \approx u_{d}(t)=\mu_{k}, \quad t \in\left[\tau_{k-1}, \tau_{k}\right), \quad k=1,2, \ldots, d,
$$

where $\mu_{k}$ is the value of the control on the $k$ th subinterval $\left[\tau_{k-1}, \tau_{k}\right)$. Define

$$
\begin{aligned}
\mathscr{U}_{d}=\left\{\mu=\left(\mu_{1}, \mu_{2}, \ldots, \mu_{d}\right)^{\top} \in \mathbb{R}^{d} \mid\right. \\
\left.R_{1} \leq \mu_{k} \leq R_{2}, k=1,2, \ldots, d\right\} .
\end{aligned}
$$

Then the approximate piecewise-constant control can be written as follows:

$$
u_{d}(t \mid \mu)=\sum_{k=1}^{d} \mu_{k} \chi_{\left[\tau_{k-1}, \tau_{k}\right)}(t), \quad t \in[0, T], \mu \in \mathcal{U}_{d},
$$

where $\chi_{\left[\tau_{k-1}, \tau_{k}\right)}$ is the characteristic function of the interval $\left[\tau_{k-1}, \tau_{k}\right), k=1,2, \ldots, d$. Substituting (37) into the dynamic system (28) yields that

$$
\begin{aligned}
\dot{Z}(t) & =G\left(t, Z(t), u_{d}(t \mid \mu)\right) \\
& =\sum_{k=1}^{d} G\left(t, Z(t), \mu_{k}\right) \chi_{\left[\tau_{k-1}, \tau_{k}\right)}(t), \quad t \in[0, T], \\
Z(0) & =Z_{0} .
\end{aligned}
$$

Let $Z_{d}(\cdot \mid \mu)$ denote the solution of system (38) corresponding to $\mu \in \mathscr{U}_{d}$. Thus, from the problem $\left(\mathrm{OCP}^{h}\right)$, we can obtain another parameter optimization problem, which is stated as follows:

$$
\left(\mathrm{OCP}_{d}^{h}\right) \min _{u \in \mathcal{U}_{d}} J_{d}^{h}(\mu)
$$

subject to (38), where

$$
\begin{aligned}
J_{d}^{h}(\mu)= & \frac{1}{2} \int_{0}^{T}\left(Z_{d}(t \mid \mu), \Pi Z_{d}(t \mid \mu)\right)_{\mathbb{R}^{4 N}} d t \\
& +\frac{1}{2} \int_{0}^{T}\left|u_{d}(t \mid \mu)\right|^{2} d t .
\end{aligned}
$$

After the parameterization of control, problem $\left(\mathrm{OCP}_{d}^{h}\right)$ involves a finite number of decision variables. Thus, it should be much easier to solve than problem $\left(\mathrm{OCP}^{h}\right)$, which involves determining the value of a function at an infinite number of time points.

\section{Variational Method for Solving Problem $\left(\mathbf{O C P}_{d}^{h}\right)$}

Problem $\left(\mathrm{OCP}_{d}^{h}\right)$ is an optimal parameter selection problem in the canonical form [24], which can be solved as nonlinear optimization problems using the SQP method. Standard SQP algorithm for nonlinear optimization exploits the gradient of the cost functional to generate search directions that lead to 
profitable areas of the search space $[25,26]$. For the approximate problem $\left(\mathrm{OCP}_{d}^{h}\right)$, the cost functional is implicit function of the decision vector $\mu$. Using the variational method [27, 28 ], we can compute this gradient and solve problem $\left(\mathrm{OCP}_{d}^{h}\right)$.

For each $m=1,2, \ldots, d$, it follows from (38) that

$$
\begin{array}{r}
Z_{d}(t \mid \mu)=Z_{d}\left(\tau_{m-1} \mid \mu\right) \\
+\int_{\tau_{m-1}}^{t} G\left(t, Z_{d}(s \mid \mu), \mu_{m}\right) d s, \\
\quad t \in\left[\tau_{m-1}, \tau_{m}\right) .
\end{array}
$$

Then for $t \in\left[\tau_{m-1}, \tau_{m}\right)$, differentiating (41) with respect to $\mu_{k}$ yields that

$$
\begin{aligned}
\frac{\partial Z_{d}(t \mid \mu)}{\partial \mu_{k}}= & \frac{\partial Z_{d}\left(\tau_{m-1} \mid \mu\right)}{\partial \mu_{k}} \\
& +\int_{\tau_{m-1}}^{t} \frac{\partial G\left(s, Z_{d}(s \mid \mu), \mu_{m}\right)}{\partial Z_{d}} \frac{\partial Z_{d}(s \mid \mu)}{\partial \mu_{k}} d s, \quad \text { for } k<m, \\
\frac{\partial Z_{d}(t \mid \mu)}{\partial \mu_{k}}= & \frac{\partial Z_{d}\left(\tau_{m-1} \mid \mu\right)}{\partial \mu_{k}} \\
& +\int_{\tau_{m-1}}^{t} \frac{\partial G\left(s, Z_{d}(s \mid \mu), \mu_{m}\right)}{\partial Z_{d}} \frac{\partial Z_{d}(s \mid \mu)}{\partial \mu_{k}} d s \\
& +\int_{\tau_{m-1}}^{t} \frac{\partial G\left(s, Z_{d}(s \mid \mu), \mu_{m}\right)}{\partial u} d s, \\
& \frac{\partial Z_{d}(t \mid \mu)}{\partial \mu_{k}}=0, \quad \text { for } k>m .
\end{aligned}
$$

Here we have

$$
\begin{aligned}
& \frac{\partial G\left(t, Z_{d}(t \mid \mu), \mu_{m}\right)}{\partial Z_{d}}=\left(\begin{array}{cc}
0 & I \\
-M^{-1} K & -\mu_{m} M^{-1} R
\end{array}\right), \\
& \frac{\partial G\left(t, Z_{d}(t \mid \mu), \mu_{m}\right)}{\partial u}=\left(\begin{array}{cc}
0 & 0 \\
0 & -M^{-1} R
\end{array}\right) Z_{d}(t \mid \mu),
\end{aligned}
$$

for $t \in\left[\tau_{m-1}, \tau_{m}\right)$. Define

$$
\begin{aligned}
& \delta_{k m}= \begin{cases}1, & \text { if } k=m, \\
0, & \text { otherwise, }\end{cases} \\
& \widehat{\delta}_{k m}= \begin{cases}1, & \text { if } k \leq m, \\
0, & \text { otherwise. }\end{cases}
\end{aligned}
$$

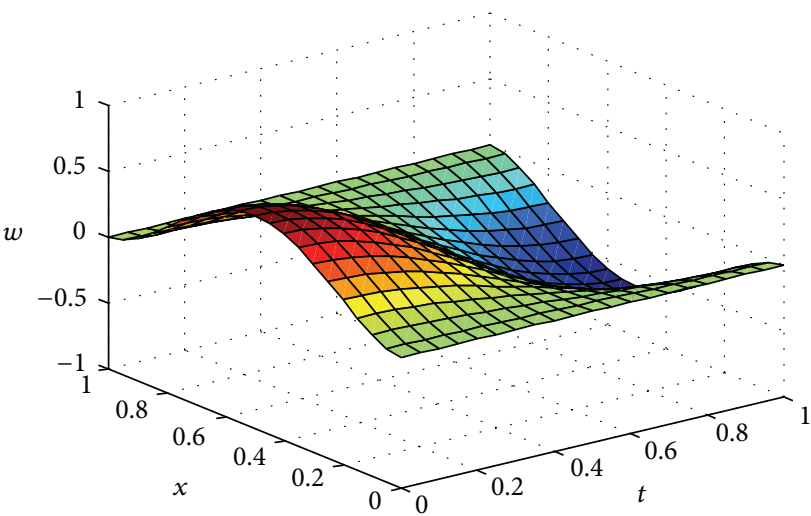

FIgURE 1: The state without damping control; that is, $u=0$.

Then, by (42) we obtain

$$
\begin{aligned}
& \frac{\partial Z_{d}(t \mid \mu)}{\partial \mu_{k}} \\
& =\widehat{\delta}_{k m} \frac{\partial Z_{d}\left(\tau_{m-1} \mid \mu\right)}{\partial \mu_{k}} \\
& \quad+\widehat{\delta}_{k m} \int_{\tau_{m-1}}^{t} \frac{\partial G\left(s, Z_{d}(s \mid \mu), \mu_{m}\right)}{\partial Z_{d}} \frac{\partial Z_{d}(s \mid \mu)}{\partial \mu_{k}} d s \\
& \quad+\delta_{k m} \int_{\tau_{m-1}}^{t} \frac{\partial G\left(s, Z_{d}(s \mid \mu), \mu_{m}\right)}{\partial u} d s, \\
& \quad t \in\left[\tau_{m-1}, \tau_{m}\right) .
\end{aligned}
$$

Moreover, it is easy to see that

$$
\frac{\partial Z_{d}(0 \mid \mu)}{\partial \mu_{k}}=0 .
$$

For $t \in\left[\tau_{m-1}, \tau_{m}\right)$, differentiating (45) with respect to $t$ yields that

$$
\begin{aligned}
\frac{d}{d t}\left\{\frac{\partial Z_{d}(t \mid \mu)}{\partial \mu_{k}}\right\}= & \widehat{\delta}_{k m} \frac{\partial G\left(t, Z_{d}(t \mid \mu), \mu_{m}\right)}{\partial Z_{d}} \frac{\partial Z_{d}(t \mid \mu)}{\partial \mu_{k}} \\
& +\delta_{k m} \frac{\partial G\left(t, Z_{d}(t \mid \mu), \mu_{m}\right)}{\partial u} .
\end{aligned}
$$

Now, we define

$$
\Gamma_{k}(t \mid \mu)=\frac{\partial Z_{d}(t \mid \mu)}{\partial \mu_{k}}, \quad k=1,2, \ldots, d .
$$

Then, it follows from (46) and (47) that

$$
\begin{aligned}
\frac{d \Gamma_{k}(t \mid \mu)}{d t}= & \widehat{\delta}_{k m} \frac{\partial G\left(t, Z_{d}(t \mid \mu), \mu_{m}\right)}{\partial Z_{d}} \Gamma_{k}(t \mid \mu) \\
& +\delta_{k m} \frac{\partial G\left(t, Z_{d}(t \mid \mu), \mu_{m}\right)}{\partial u}, \\
& t \in\left[\tau_{m-1}, \tau_{m}\right), \quad m=1,2, \ldots, d,
\end{aligned}
$$



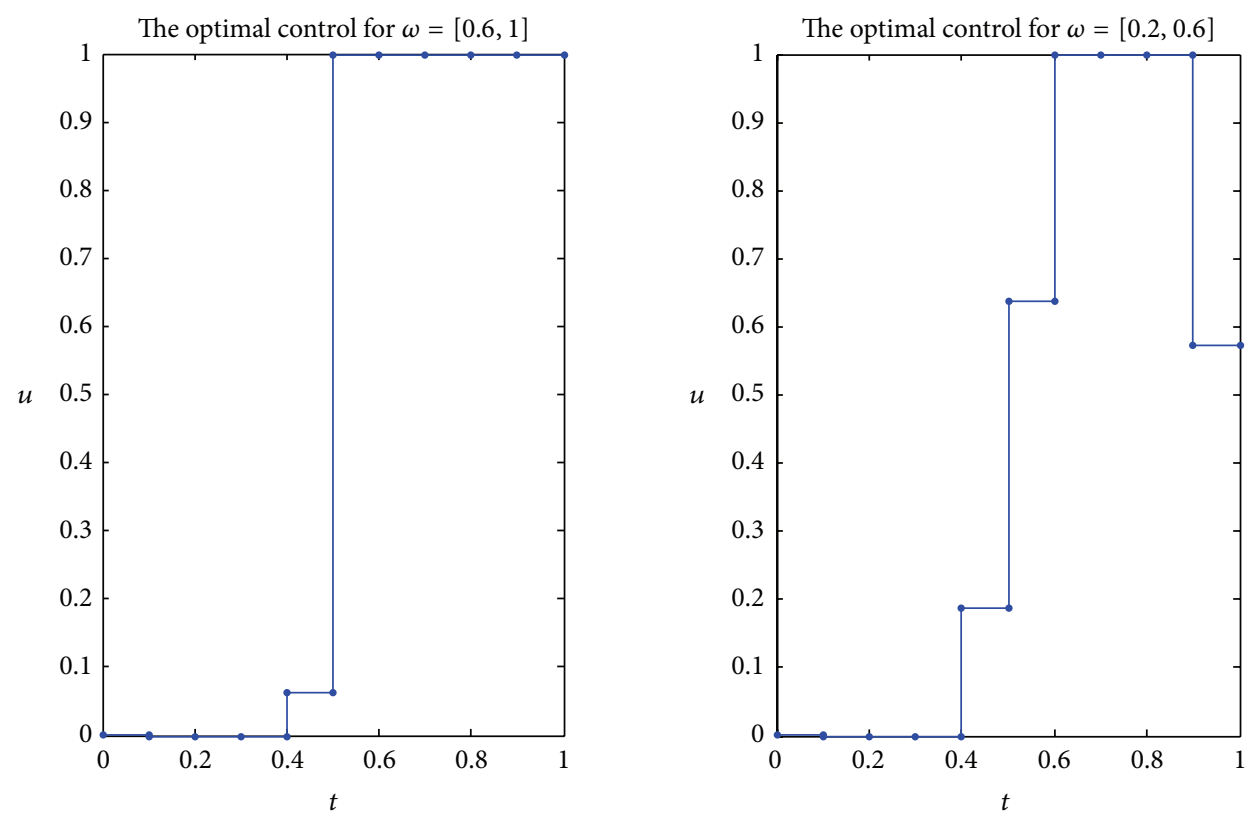

Figure 2: The optimal controls for $(\mathrm{OCP})_{1}$ and $(\mathrm{OCP})_{2}$.

with the initial condition

$$
\Gamma_{k}(0 \mid \mu)=0 .
$$

As a result, by using the chain rule, we can derive the gradient of $J_{d}^{h}(\mu)$ with respect to $\mu_{k}, k=1,2, \ldots, d$, as follows:

$$
\begin{aligned}
& \frac{\partial J_{d}^{h}(\mu)}{\partial \mu_{k}} \\
& \quad=\int_{0}^{T}\left(Z_{d}(t \mid \mu), \Pi \Gamma_{k}(t \mid \mu)\right)_{\mathbb{R}^{4 N}} d t+\mu_{k}\left(\tau_{k}-\tau_{k-1}\right) .
\end{aligned}
$$

By incorporating these formulae into the SQP algorithm, we can solve the problem $\left(\mathrm{OCP}_{d}^{h}\right)$ numerically.

\section{Numerical Simulations}

In this section, we present some numerical simulation results by the approximation method presented in this paper. Let $L=$ $T=1, \rho(x)=q(x)=1, R_{1}=0$, and $R_{2}=1$. Moreover, we take

$$
\begin{gathered}
w_{0}(x)=10 x^{2}(1-x)^{2}, \quad w_{1}(x)=0, \\
f(x, t)=\left(240-10 \pi^{2} x^{2}(1-x)^{2}\right) \cos (\pi t) .
\end{gathered}
$$

If the damping coefficient

$$
D(x, t)=0, \quad(x, t) \in Q,
$$

which implies that the system (1) is without any damping, the exact solution (see Figure 1) of (1) is

$$
w(x, t)=10 \cos (\pi t) x^{2}(1-x)^{2}, \quad(x, t) \in Q .
$$

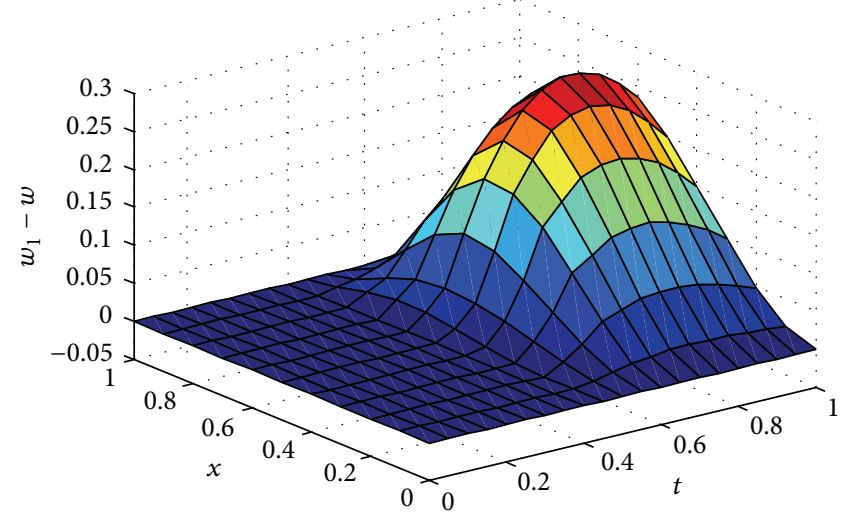

FIgURE 3: The difference between $w_{1}$ and $w$.

In this case, we have

$$
\frac{1}{2} \int_{0}^{T}\left\|\left(\begin{array}{c}
w(t) \\
\dot{w}(t)
\end{array}\right)\right\|_{\mathscr{H}}^{2} d t+\frac{1}{2} \int_{0}^{1}|u(t)|^{2} d t=20.39 .
$$

In the following, we discuss the optimal control problem (OCP) with local Kelvin-Voigt damping acting on two different locations. We write $\omega_{1}=[0.6,1]$ and $\omega_{2}=[0.2,0.6]$. By the approximation method presented in this paper, we can get the numerical results for the optimal control problem (OCP) with $\omega=\omega_{1}$ and $\omega=\omega_{2}$, which will be called problem $(\mathrm{OCP})_{1}$ and problem $(\mathrm{OCP})_{2}$, respectively. The numerical optimal controls for the two problems are presented by Figure 2. Moreover, the difference of $w_{1}$ and $w$ is shown in Figure 3, where $w$ is defined by (54) and $w_{1}$ is the numerical state function of (OCP $)_{1}$. The difference of $w_{2}$ and $w$ has the same property, where $w_{2}$ is the numerical state function of $(\mathrm{OCP})_{2}$. For problem $(\mathrm{OCP})_{1}$, the optimal value is 14.22 and 
for problem $(\mathrm{OCP})_{2}$, the optimal value is 15.65 , which means that the control effect of the location $\omega_{1}$ is better than the location $\omega_{2}$.

\section{Conflict of Interests}

The authors declare that there is no conflict of interests regarding the publication of this paper.

\section{Acknowledgments}

This work was partially supported by the National Natural Science Foundation of China (61374096 and 61104048) and the Natural Science Foundation of Zhejiang (Y6110751).

\section{References}

[1] H. T. Banks, R. C. Smith, and Y. Wang, "The modeling of piezoceramic patch interactions with shells, plates, and beams," Quarterly of Applied Mathematics, vol. 53, no. 2, pp. 353-381, 1995.

[2] H. T. Banks and Y. Zhang, "Computational methods for a curved beam with piezoceramic patches," Journal of Intelligent Material Systems and Structures, vol. 8, no. 3, pp. 260-278, 1997.

[3] K. H. Ip and P. C. Tse, "Optimal configuration of a piezoelectric patch for vibration control of isotropic rectangular plates," Smart Materials and Structures, vol. 10, no. 2, pp. 395-403, 2001.

[4] S. J. Dyke, B. F. Spencer Jr., M. K. Sain, and J. D. Carlson, “Modeling and control of magnetorheological dampers for seismic response reduction," Smart Materials and Structures, vol. 5, no. 5, pp. 565-575, 1996.

[5] Y. Kim, R. Langari, and S. Hurlebaus, "Semiactive nonlinear control of a building with a magnetorheological damper system," Mechanical Systems and Signal Processing, vol. 23, no. 2, pp. 300-315, 2009.

[6] C. Y. Lai and W. H. Liao, "Vibration control of a suspension system via a magnetorheological fluid damper," Journal of Vibration and Control, vol. 8, no. 4, pp. 527-547, 2002.

[7] G. Yang, B. F. Spencer Jr., H. J. Jung, and J. D. Carlson, "Dynamic modeling of large-scale magnetorheological damper systems for civil engineering applications," Journal of Engineering Mechanics, vol. 130, no. 9, pp. 1107-1114, 2004.

[8] Z. Y. Liu and S. M. Zheng, Semigroups Associated with Dissipative Systems, vol. 398, CRC Press, New York, NY, USA, 1999.

[9] C. Zhang, "Boundary feedback stabilization of the undamped Timoshenko beam with both ends free," Journal of Mathematical Analysis and Applications, vol. 326, no. 1, pp. 488-499, 2007.

[10] H. L. Zhao, K. S. Liu, and C. G. Zhang, "Stability for the Timoshenko beam system with local Kelvin-Voigt damping," Acta Mathematica Sinica (English Series), vol. 21, no. 3, pp. 655666, 2005.

[11] K. S. Liu and Z. Y. Liu, "Exponential decay of energy of the Euler-Bernoulli beam with locally distributed Kelvin-Voigt damping," SIAM Journal on Control and Optimization, vol. 36, no. 3, pp. 1086-1098, 1998.

[12] B. Z. Guo, "Riesz basis property and exponential stability of controlled Euler-Bernoulli beam equations with variable coefficients," SIAM Journal on Control and Optimization, vol. 40, no. 6, pp. 1905-1923, 2002.
[13] K. Ammari and M. Tucsnak, "Stabilization of Bernoulli-Euler beams by means of a pointwise feedback force," SIAM Journal on Control and Optimization, vol. 39, no. 4, pp. 1160-1181, 2000.

[14] G. Chen, S. G. Krantz, D. W. Ma, and C. E. Wayne, “The EulerBernoulli beam equation with boundary energy dissipation," in Operator Methods for Optimal control Problems, S. J. Lee, Ed., vol. 108 of Lecture Notes in Pure and Appl. Math., pp. 67-96, Louisiana, Baton Rouge, La, USA, 1987.

[15] B. Guo and K. Yang, "Dynamic stabilization of an EulerBernoulli beam equation with time delay in boundary observation," Automatica, vol. 45, no. 6, pp. 1468-1475, 2009.

[16] I. Y. Shen, "Stability and controllability of Euler-Bernoulli beams with intelligent constrained layer treatments," Journal of Vibration and Acoustics, vol. 118, no. 1, pp. 70-77, 1996.

[17] N. Tanaka and Y. Kikushima, "Optimal vibration feedback control of an Euler-Bernoulli beam: toward realization of the active sink method," Journal of Vibration and Acoustics, Transactions of the ASME, vol. 121, no. 2, pp. 174-182, 1999.

[18] J. M. Ball, J. E. Marsden, and M. Slemrod, "Controllability for distributed bilinear systems," SIAM Journal on Control and Optimization, vol. 20, no. 4, pp. 575-597, 1982.

[19] K. Beauchard, "Local controllability and non-controllability for a 1D wave equation with bilinear control," Journal of Differential Equations, vol. 250, no. 4, pp. 2064-2098, 2011.

[20] M. Slemrod, "Stabilization of bilinear control systems with applications to nonconservative problems in elasticity," SIAM Journal on Control and Optimization, vol. 16, no. 1, pp. 131-141, 1978.

[21] Q. Lin, R. Loxton, and K. L. Teo, “The control parameterization method for nonlinear optimal control: a survey," Journal of Industrial and Management Optimization, vol. 10, no. 1, pp. 275309, 2014.

[22] Q. Lin, R. Loxton, K. L. Teo, and Y. H. Wu, "Optimal control computation for nonlinear systems with state-dependent stopping criteria," Automatica, vol. 48, no. 9, pp. 2116-2129, 2012.

[23] R. C. Loxton, K. L. Teo, V. Rehbock, and K. F. Yiu, "Optimal control problems with a continuous inequality constraint on the state and the control," Automatica, vol. 45, no. 10, pp. 2250-2257, 2009.

[24] K. L. Teo, C. J. Goh, and K. H. Wong, A Unified Computational Approach to Optimal Control Problems, Longman Scientific and Technical, London, UK, 1991.

[25] M. S. Bazaraa, H. D. Sherali, and C. M. Shetty, Nonlinear Programming: Theory and Algorithms, John Wiley \& Sons, New York, NY, USA, 2013.

[26] D. G. Luenberger and Y. Y. Ye, Linear and Nonlinear Programming, vol. 116, Springer, New York, NY, USA, 2008.

[27] R. C. Loxton, K. L. Teo, and V. Rehbock, "Optimal control problems with multiple characteristic time points in the objective and constraints," Automatica, vol. 44, no. 11, pp. 2923-2929, 2008.

[28] R. Loxton, K. L. Teo, and V. Rehbock, "Robust suboptimal control of nonlinear systems," Applied Mathematics and Computation, vol. 217, no. 14, pp. 6566-6576, 2011. 


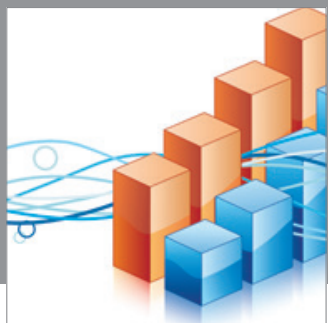

Advances in

Operations Research

mansans

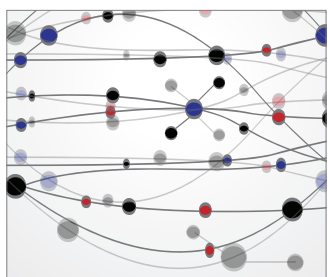

The Scientific World Journal
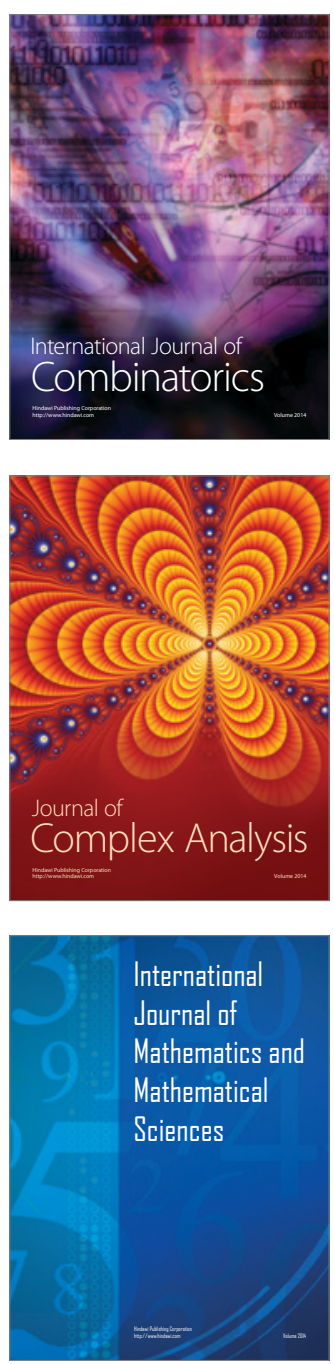
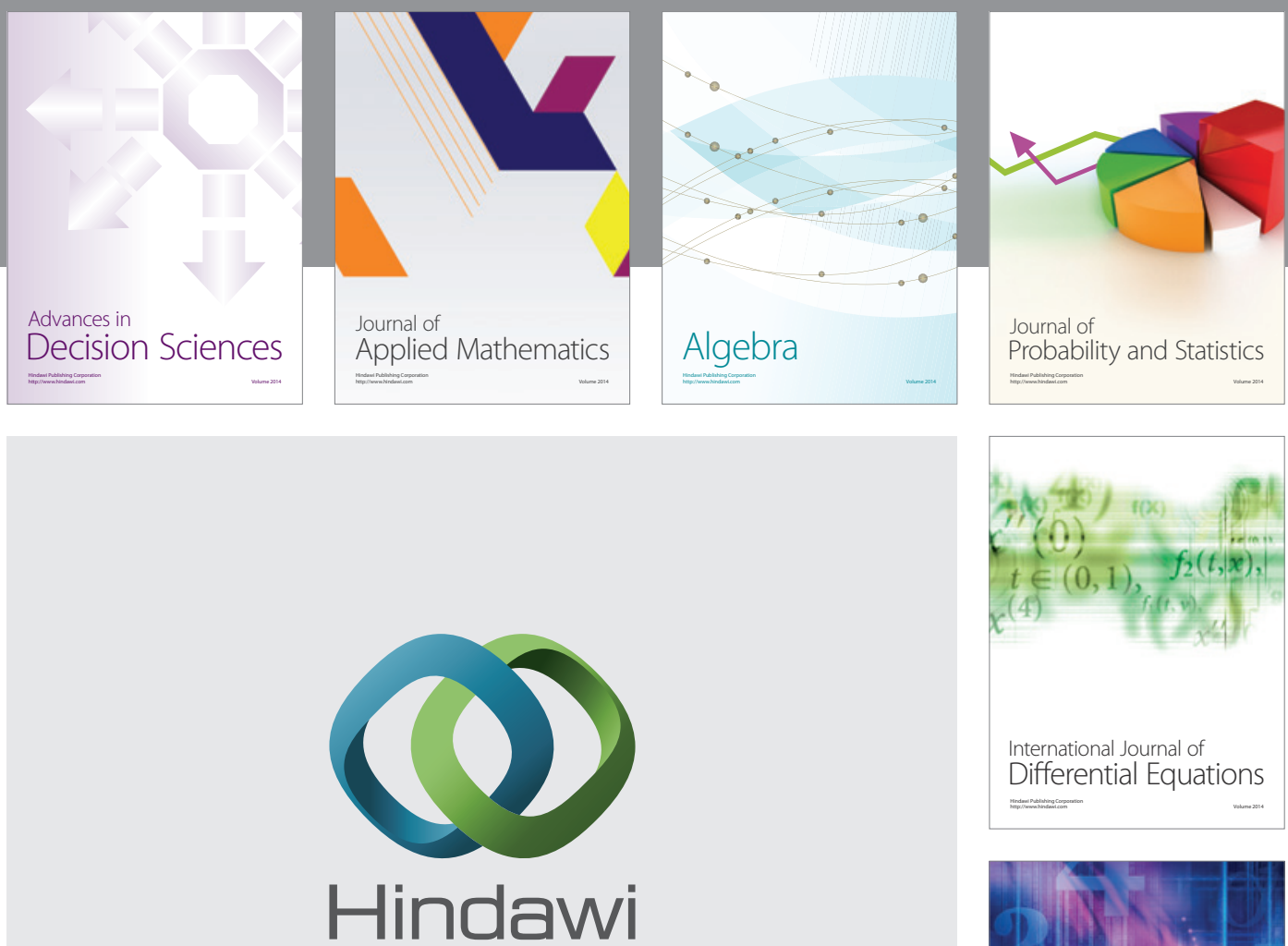

Submit your manuscripts at http://www.hindawi.com
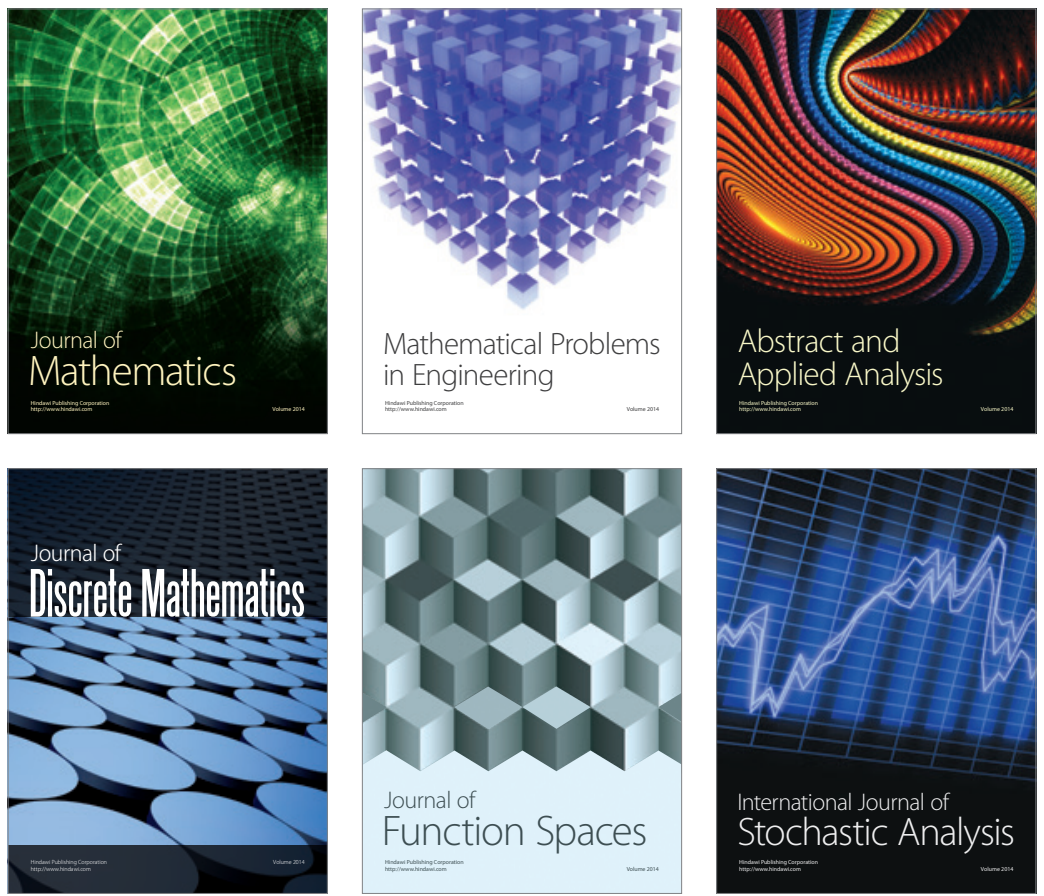

Journal of

Function Spaces

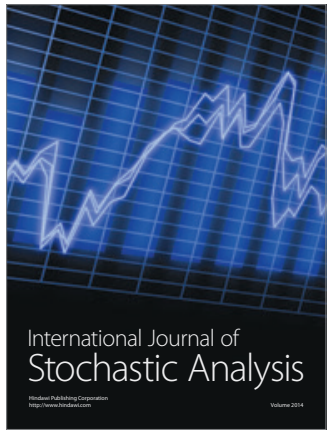

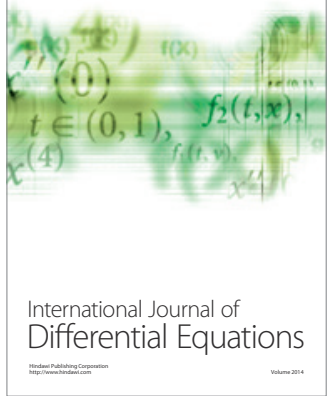
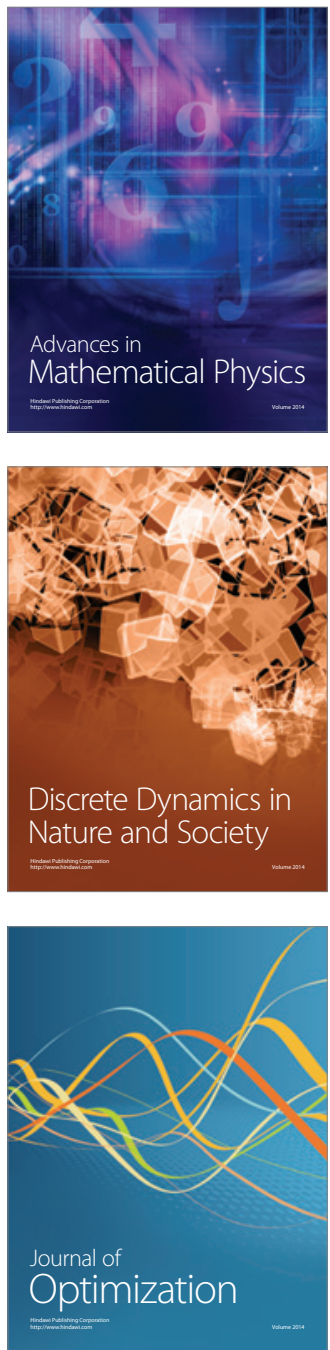